Document downloaded from:

http://hdl.handle.net/10251/56199

This paper must be cited as:

Babajee, DKR.; Cordero Barbero, A.; Soleymani, F.; Torregrosa Sánchez, JR. (2014). On improved three-step schemes with high efficiency index and their dynamics. Numerical Algorithms. 65(1):153-169. doi:10.1007/s11075-013-9699-6.

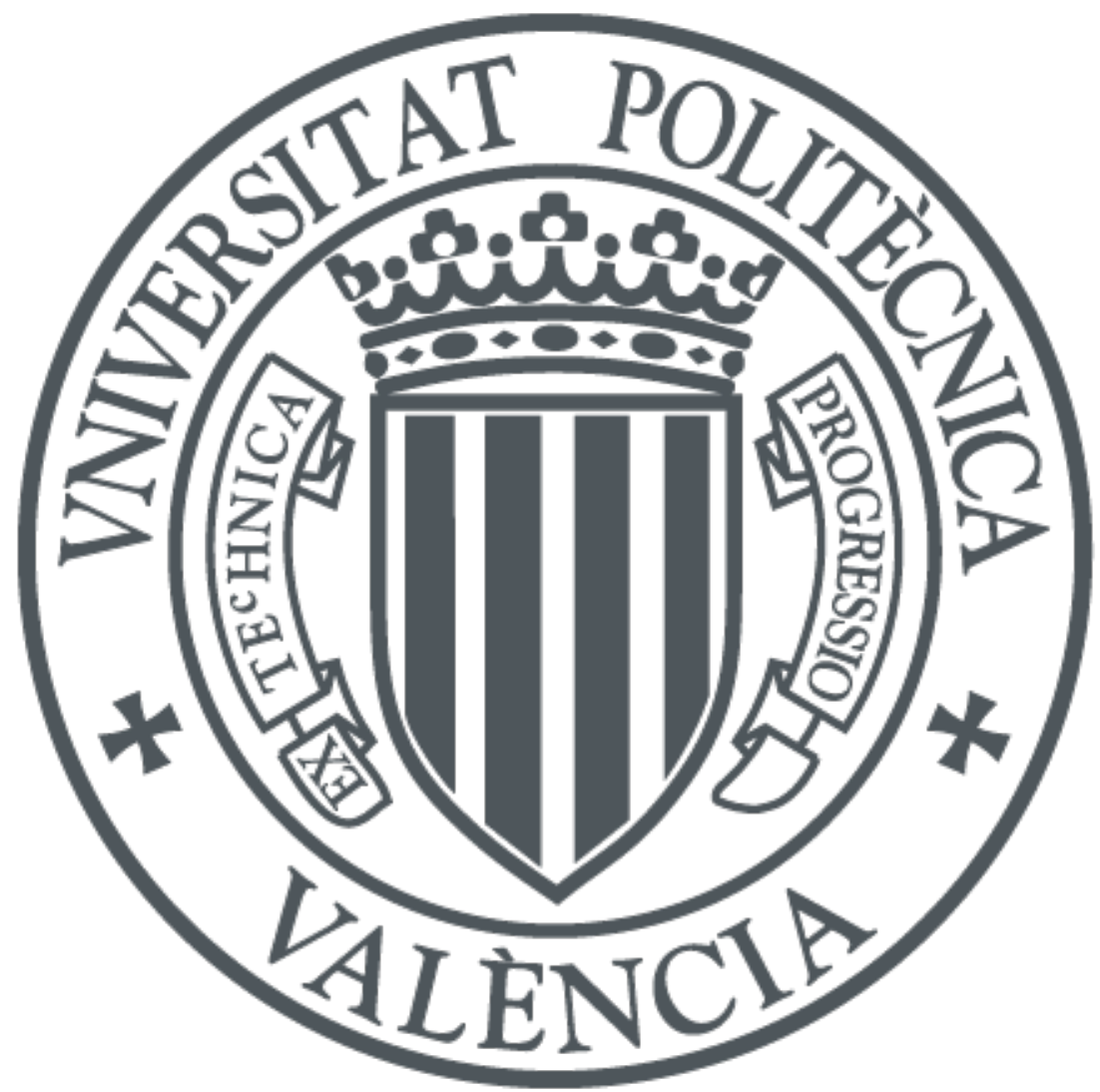

The final publication is available at

http://dx.doi.org/10.1007/s11075-013-9699-6

Copyright Springer Verlag

Additional Information 


\title{
On Improved Three-Step Schemes with High Efficiency Index and Their Dynamics
}

\author{
Diyashvir K.R. Babajee • Alicia \\ Cordero - Fazlollah Soleymani - Juan R. \\ Torregrosa
}

Received: date / Accepted: date

\begin{abstract}
This paper presents an improvement of the sixth-order method of Chun and Neta as a class of three-step iterations with optimal efficiency index, in the sense of Kung-Traub conjecture. Each member of the presented class reaches the highest possible order using four functional evaluations. Error analysis will be studied and numerical examples are also made to support the theoretical results. We then present results which describe the dynamics of the presented optimal methods for complex polynomials. The basins of attraction of the existing optimal methods and our methods are presented and compared to illustrate their performances.
\end{abstract}

Keywords Basin of attraction $\cdot$ Kung-Traub conjecture $\cdot$ fractal $\cdot$ multi-point iterations · Julia set

This research was supported by Ministerio de Ciencia y Tecnología MTM2011-28636-C02-02 and FONDOCYT República Dominicana. The first author is an IEEE member, Mauritius Subsection.

Alicia Cordero, Juan R. Torregrosa

Instituto de Matemática Multidisciplinar,

Universidad Politécnica de Valencia,

Camino de Vera, s/n, 46022

Valencia, Spain

Diyashvir K.R. Babajee

Allied Network for Policy Research \& Advocacy for Sustainability,

Mauritius, IEEE Subsection, Mauritius

Fazlollah Soleymani

Department of Mathematics, Islamic Azad University,

Zahedan Branch, PO Box 987-98138 Zahedan, Iran 


\section{Introduction}

Nonlinear equations arise in many real problems in Science and Engineering. The subject of root-finding of nonlinear equations play an important role in Numerical Analysis and Optimization. In particular, Newton's scheme and its modifications can be used to solve the variational inequality and the nonlinear complementary problems (see, for example [1] and [2]). Also efficient iterative schemes are needed to solve nonlinear equations coming from the application of shooting methods to solve boundary value problems.

Throughout this paper we consider iterative methods to find a simple root $\alpha$ in an open interval $D$, i.e., $f(\alpha)=0$ and $f^{\prime}(\alpha) \neq 0$, of a nonlinear equation $f(x)=0$.

Recenly, Chun and Neta in [3] presented a sixth-order method as follows

$$
\left\{\begin{array}{l}
y_{n}=x_{n}-\frac{f\left(x_{n}\right)}{f^{\prime}\left(x_{n}\right)}, \\
z_{n}=y_{n}-\frac{f\left(y_{n}\right)}{f^{\prime}\left(x_{n}\right)}\left(1-\frac{f\left(y_{n}\right)}{f\left(x_{n}\right)}\right)^{-2}, \\
x_{n+1}=z_{n}-\frac{f\left(z_{n}\right)}{f^{\prime}\left(x_{n}\right)}\left(1-\frac{f\left(y_{n}\right)}{f\left(x_{n}\right)}-\frac{f\left(z_{n}\right)}{f\left(x_{n}\right)}\right)^{-2} .
\end{array}\right.
$$

This scheme without memory includes three evaluations of the function and one evaluation of the first-order derivative per computing step to reach the order six and thus its classical efficiency index is $6^{\frac{1}{4}} \approx 1.565$, which is not as high as possible with four functional evaluations according to the hypothesis of Kung and Traub on the optimality of multi-point iterations without memory [4].

To improve the local order of convergence different techniques have been used getting iterative schemes with and without memory (see, for instance [5-7]) and to upgrade the efficiency index, many optimal methods without memory have been proposed; see, for example, [3,8-16], and the references therein.

The prime motivation of this paper is to optimalize (1) in terms of the local convergence order and the efficiency index, as well as to study the dynamics of some optimal eighth-order methods using fractal picture. Thus, we aim at modifying (1) to reach a wide class of three-step methods using four evaluations, i.e. the same as (1), but with higher order of convergence and efficiency. This purpose will be studies by using weight function technique.

The dynamical study of the rational function associated to an iterative method gives important information about the convergence and stability of the scheme. In this sense, we should not forget the early work [17] and more recent studies, as for example [18-23] and the references therein). In order to do this, we recall some concepts. Thus, now we shortly present them. Let $R: \hat{\mathbb{C}} \rightarrow \widehat{\mathbb{C}}$ be a rational map on the Riemann sphere. For $z \in \hat{\mathbb{C}}$, we define its orbit as the set $\operatorname{orb}(z)=\left\{z, R(z), R^{2}(z), \cdots, R^{n}(z), \cdots\right\}$, and subsequently, a point $z_{0}$ is a fixed point of $R$ if $R\left(z_{0}\right)=z_{0}$.

A periodic point $z_{0}$ of period $m$ is such that $R^{m}\left(z_{0}\right)=z_{0}$ where $m$ is the smallest such integer. And also a point $z_{0}$ is called attracting if $\left|R^{\prime}\left(z_{0}\right)\right|<1$, 
repelling if $\left|R^{\prime}\left(z_{0}\right)\right|>1$, and neutral if $\left|R^{\prime}\left(z_{0}\right)\right|=1$. The Julia set of a nonlinear map $R(z)$, denoted $\mathcal{J}(R)$, is the closure of the set of its repelling periodic points. The complement of $\mathcal{J}(R)$ is the Fatou set $\mathcal{F}(R)$, where the basin of attraction of the different roots lie.

The rest of the paper is organized in what follows. In the next section, we derive a new family of three-step eighth-order convergent methods, which is consistent with the Kung-Traub conjecture. Section 3 covers some concrete optimal eighth order of convergence methods of the class. Next, in Section 4, we give a number of numerical tests to clarify the effectiveness of the new attained methods. Section 5 successfully discusses the dynamics of the presented optimal iterations. The concluding remarks of the paper is furnished in Section 6 .

\section{The improved scheme}

To build an optimal eighth-order method consuming four evaluations according to (1), we suggest the following iterative class by using weight function approach at the end of the first and third steps

$$
\left\{\begin{array}{l}
y_{n}=x_{n}-\frac{f\left(x_{n}\right)}{f^{\prime}\left(x_{n}\right)} A(\delta) \\
z_{n}=y_{n}-\frac{f\left(y_{n}\right)}{f^{\prime}\left(x_{n}\right)}\left(1-\frac{f\left(y_{n}\right)}{f\left(x_{n}\right)}\right)^{-2} \\
x_{n+1}=z_{n}-\frac{f\left(z_{n}\right)}{f^{\prime}\left(x_{n}\right)}\left(1-\frac{f\left(y_{n}\right)}{f\left(x_{n}\right)}-\frac{f\left(z_{n}\right)}{f\left(x_{n}\right)}\right)^{-2}\{G(t)+H(\mu)\}
\end{array}\right.
$$

where $\delta=\frac{f(x)}{f^{\prime}(x)}, t=\frac{f(y)}{f(x)}$, and $\mu=\frac{f(z)}{f(y)}$, without the index $n$. The three weight functions $A(\delta), G(t)$, and $H(\mu)$, should be chosen such that the order arrives at local eight. This is done and illustrated in Theorem 1.

Theorem 1 Let $\alpha \in D$ be a simple zero of a sufficiently differentiable function $f: D \subseteq \mathbb{R} \rightarrow \mathbb{R}$ for an open interval $D$, which contains $x_{0}$ as an initial approximation of $\alpha$. Then the three-step iteration (2) without memory, which contains four evaluations per full cycle has the optimal convergence rate eight and satisfies the error equation below

$$
\begin{aligned}
e_{n+1}= & \frac{1}{24 c_{1}^{7}} c_{2}\left(-2 c_{2}^{2}+c_{1} c_{3}\right)\left(24 c_{1} c_{2}^{2} c_{3}\left(7-2 H^{\prime \prime}(0)\right)+12 c_{1}^{2} c_{3}^{2}\left(-2+H^{\prime \prime}(0)\right)\right. \\
& \left.+12 c_{1}^{2} c_{2}\left(-2 c_{4}+c_{1} A^{(3)}(0)\right)+c_{2}^{4}\left(-120+48 H^{\prime \prime}(0)+G^{(4)}(0)\right)\right) e_{n}^{8} \\
& +O\left(e_{n}^{9}\right)
\end{aligned}
$$

when $A(0)=1, A^{\prime}(0)=0, A^{\prime \prime}(0)=0,\left|A^{(3)}(0)\right|<\infty,|G(0)|<\infty, G^{\prime}(0)=0$, $G^{\prime \prime}(0)=2, G^{(3)}(0)=0,\left|G^{(4)}(0)\right|<\infty, H(0)=1-G(0), H^{\prime}(0)=1$ and $\left|H^{\prime \prime}(0)\right|<\infty$. 
Proof. By defining $e_{n}=x_{n}-\alpha$ as the error of the iterative scheme in each iteration, applying the Taylor's series expansion and taking into account $f(\alpha)=0$, we have

$$
f\left(x_{n}\right)=c_{1} e_{n}+c_{2} e_{n}^{2}+c_{3} e_{n}^{3}+c_{4} e_{n}^{4}+c_{5} e_{n}^{5}+c_{6} e_{n}^{6}+c_{7} e_{n}^{7}+c_{8} e_{n}^{8}+O\left(e_{n}^{9}\right),
$$

where $c_{k}=\frac{f^{(k)}(\alpha)}{k !}, k \geq 1$.

Furthermore, we have

$$
f^{\prime}\left(x_{n}\right)=c_{1}+2 c_{2} e_{n}+3 c_{3} e_{n}^{2}+4 c_{4} e_{n}^{3}+5 c_{5} e_{n}^{4}+6 c_{6} e_{n}^{5}+7 c_{7} e_{n}^{6}+8 c_{8} e_{n}^{7}+O\left(e_{n}^{8}\right) \text {. }
$$

Dividing (4) by (5) gives us

$$
\begin{aligned}
\frac{f\left(x_{n}\right)}{f^{\prime}\left(x_{n}\right)}= & e_{n}-\frac{c_{2} e_{n}^{2}}{c_{1}}+\frac{2\left(c_{2}^{2}-c_{1} c_{3}\right) e_{n}^{3}}{c_{1}^{2}} \\
& +\frac{\left(-4 c_{2}^{3}+7 c_{1} c_{2} c_{3}-3 c_{1}^{2} c_{4}\right) e_{n}^{4}}{c_{1}^{3}}+\cdots+O\left(e_{n}^{8}\right) .
\end{aligned}
$$

Substituting (6) in $y_{n}$ and writing the Taylor's expansion for $f\left(y_{n}\right)$, we obtain by considering $A(0)=1, A^{\prime}(0)=0, A^{\prime \prime}(0)=0$, and $\left|A^{(3)}(0)\right|<\infty$, respectively

$$
\begin{aligned}
y_{n}=\alpha & +\frac{c_{2} e_{n}^{2}}{c_{1}}+\frac{2\left(-c_{2}^{2}+c_{1} c_{3}\right) e_{n}^{3}}{c_{1}^{2}} \\
& +\left(\frac{4 c_{2}^{3}-7 c_{1} c_{2} c_{3}+3 c_{1}^{2} c_{4}}{c_{1}^{3}}-\frac{1}{6} A^{(3)}(0)\right) e_{n}^{4}+\cdots+O\left(e_{n}^{8}\right), \\
f\left(y_{n}\right)= & c_{2} e_{n}^{2}+\left(-\frac{2 c_{2}^{2}}{c_{1}}+2 c_{3}\right) e_{n}^{3} \\
& +\left(\frac{5 c_{2}^{3}}{c_{1}^{2}}-\frac{7 c_{2} c_{3}}{c_{1}}+3 c_{4}-\frac{1}{6} c_{1} A^{(3)}(0)\right) e_{n}^{4}+\cdots+O\left(e_{n}^{8}\right) .
\end{aligned}
$$

Moreover for the second step of (2), we find

$$
\begin{aligned}
z_{n}-\alpha= & \frac{\left(2 c_{2}^{3}-c_{1} c_{2} c_{3}\right) e_{n}^{4}}{c_{1}^{3}}+\frac{\left(-6\left(5 c_{2}^{4}-7 c_{1} c_{2}^{2} c_{3}+c_{1}^{2} c_{3}^{2}+c_{1}^{2} c_{2} c_{4}\right)+c_{1}^{3} c_{2} A^{(3)}(0)\right) e_{n}^{5}}{3 c_{1}^{4}} \\
& +\frac{1}{12 c_{1}^{5}}\left(372 c_{2}^{5}-864 c_{1} c_{2}^{3} c_{3}+2 c_{1}^{2} c_{2}^{2}\left(126 c_{4}-13 c_{1} A^{(3)}(0)\right)\right. \\
& \left.+2 c_{1}^{3} c_{3}\left(-42 c_{4}+5 c_{1} A^{(3)}(0)\right)+c_{1}^{2} c_{2}\left(360 c_{3}^{2}+c_{1}\left(-36 c_{5}+c_{1} A^{(4)}(0)\right)\right)\right) e_{n}^{6} \\
& +\cdots+O\left(e_{n}^{9}\right) .
\end{aligned}
$$

Using (9), we now have

$$
\begin{aligned}
f\left(z_{n}\right)= & \frac{\left(2 c_{2}^{3}-c_{1} c_{2} c_{3}\right) e_{n}^{4}}{c_{1}^{2}}+\left(-\frac{2\left(5 c_{2}^{4}-7 c_{1} c_{2}^{2} c_{3}+c_{1}^{2} c_{3}^{2}+c_{1}^{2} c_{2} c_{4}\right)}{c_{1}^{3}}+\frac{1}{3} c_{2} A^{(3)}(0)\right) e_{n}^{5} \\
& +\frac{1}{12 c_{1}^{4}}\left(372 c_{2}^{5}-864 c_{1} c_{2}^{3} c_{3}+2 c_{1}^{2} c_{2}^{2}\left(126 c_{4}-13 c_{1} A^{(3)}(0)\right)\right. \\
& \left.+2 c_{1}^{3} c_{3}\left(-42 c_{4}+5 c_{1} A^{(3)}(0)\right)+c_{1}^{2} c_{2}\left(360 c_{3}^{2}+c_{1}\left(-36 c_{5}+c_{1} A^{(4)}(0)\right)\right)\right) e_{n}^{6} \\
& +\cdots+O\left(e_{n}^{9}\right) .
\end{aligned}
$$


It is now easy to find

$$
\begin{aligned}
1- & \frac{f\left(y_{n}\right)}{f\left(x_{n}\right)}-\frac{f\left(z_{n}\right)}{f\left(x_{n}\right)}=1-\frac{2 c_{2} e_{n}}{c_{1}}+\frac{\left(7 c_{2}^{2}-4 c_{1} c_{3}\right) e_{n}^{2}}{c_{1}^{2}} \\
& +\left(\frac{-26 c_{2}^{3}+26 c_{1} c_{2} c_{3}-6 c_{1}^{2} c_{4}}{c_{1}^{3}}+\frac{1}{3} A^{(3)}(0)\right) e_{n}^{3} \\
& +\left(\frac{93 c_{2}^{4}}{c_{1}^{4}}-\frac{138 c_{2}^{2} c_{3}}{c_{1}^{3}}+\frac{24 c_{3}^{2}+38 c_{2} c_{4}}{c_{1}^{2}}-\frac{8\left(3 c_{5}+c_{2} A^{(3)}(0)\right)}{3 c_{1}}+\frac{1}{12} A^{(4)}(0)\right) e_{n}^{4} \\
& +\frac{1}{60 c_{1}^{5}}\left(4 0 \left(-3\left(153 c_{2}^{5}-312 c_{1} c_{2}^{3} c_{3}+99 c_{1}^{2} c_{2}^{2} c_{4}+5 c_{1}^{2} c_{2}\left(24 c_{3}^{2}-5 c_{1} c_{5}\right)\right.\right.\right. \\
& \left.\left.+5 c_{1}^{3}\left(-7 c_{3} c_{4}+c_{1} c_{6}\right)\right)+c_{1}^{3}\left(23 c_{2}^{2}-8 c_{1} c_{3}\right) A^{(3)}(0)\right)-45 c_{1}^{4} c_{2} A^{(4)}(0) \\
& \left.+c_{1}^{5} A^{(5)}(0)\right) e_{n}^{5}+O\left(e_{n}^{6}\right) .
\end{aligned}
$$

For the last step of (2), we obtain by using (7)-(10)

$$
\begin{aligned}
& e_{n+1}=\frac{c_{2}\left(-2 c_{2}^{2}+c_{1} c_{3}\right)(-1+G(0)+H(0)) e_{n}^{4}}{c_{1}^{3}} \\
& +\frac{\left(6\left(5 c_{2}^{4}-7 c_{1} c_{2}^{2} c_{3}+c_{1}^{2} c_{3}^{2}+c_{1}^{2} c_{2} c_{4}\right)(-1+G(0)+H(0))\right.}{3 c_{1}^{4}} e_{n}^{5} \\
& +\frac{\left.3 c_{2}^{2}\left(-2 c_{2}^{2}+c_{1} c_{3}\right) G^{\prime}(0)-c_{1}^{3} c_{2}(-1+G(0)+H(0)) A^{(3)}(0)\right)}{3 c_{1}^{4}} e_{n}^{5} \\
& +\frac{1}{12 c_{1}^{5}}\left(-12 c_{2}^{5}\left(-31+25 G(0)+25 H(0)-16 G^{\prime}(0)+4 H^{\prime}(0)+G^{\prime \prime}(0)\right)\right. \\
& +6 c_{1} c_{2}^{3} c_{3}\left(-144+134 G(0)+134 H(0)-42 G^{\prime}(0)+8 H^{\prime}(0)+G^{\prime \prime}(0)\right) \\
& +2 c_{1}^{3} c_{3}(-1+G(0)+H(0))\left(42 c_{4}-5 c_{1} A^{(3)}(0)\right) \\
& +2 c_{1}^{2} c_{2}^{2}\left(6 c_{4}\left(-21(-1+G(0)+H(0))+2 G^{\prime}(0)\right)\right. \\
& \left.+c_{1}\left(13(-1+G(0)+H(0))-2 G^{\prime}(0)\right) A^{(3)}(0)\right)-c_{1}^{2} c_{2}\left(12 c_{3}^{2}(-30+29 G(0)\right. \\
& \left.\left.\left.+29 H(0)-4 G^{\prime}(0)+H^{\prime}(0)\right)+c_{1}(-1+G(0)+H(0))\left(-36 c_{5}+c_{1} A^{(4)}(0)\right)\right)\right) e_{n}^{6} \\
& +\frac{1}{360 c_{1}^{6}}\left(-60 c_{1}^{2} c_{2}^{3}\left(-6 c_{4}\left(-100+90 G(0)+90 H(0)-33 G^{\prime}(0)\right.\right.\right. \\
& \left.\left.+8 H^{\prime}(0)+G^{\prime \prime}(0)\right)+c_{1}\left(-56+46 G(0)+46 H(0)-21 G^{\prime}(0)+8 H^{\prime}(0)+G^{\prime \prime}(0)\right) A^{(3)}(0)\right) \\
& +120 c_{2}^{6}\left(-222+60 G(0)+60 H(0)-213 G^{\prime}(0)+96 H^{\prime}(0)+33 G^{\prime \prime}(0)-G^{(3)}(0)\right) \\
& -60 c_{1} c_{2}^{4} c_{3}\left(-1476+948 G(0)+948 H(0)-942 G^{\prime}(0)+360 H^{\prime}(0)+84 G^{\prime \prime}(0)\right. \\
& \left.-G^{(3)}(0)\right)+5 c_{1}^{3}\left(-144 c_{3}^{3}\left(9 G(0)+9 H(0)-2\left(5+G^{\prime}(0)\right)+H^{\prime}(0)\right)+4 c_{1}(-1+G(0)+H(0))\right. \\
& \left(-18 c_{4}+c_{1} A^{(3)}(0)\right)\left(-6 c_{4}+c_{1} A^{(3)}(0)\right)-15 c_{1} c_{3}(-1+G(0)+H(0)) \\
& \left.\left(-48 c_{5}+c_{1} A^{(4)}(0)\right)\right)+15 c_{1}^{2} c_{2}^{2}\left(24 c_{3}^{2}(-188+156 G(0)+156 H(0)\right. \\
& \left.-73 G^{\prime}(0)+26 H^{\prime}(0)+3 G^{\prime \prime}(0)\right)+c_{1}\left(-672 c_{5}(-1+G(0)+H(0))\right) \\
& \left.+72 c_{5} G^{\prime}\left(0+c_{1}\left(15(-1+G(0)+H(0))-2 G^{\prime}(0)\right) A^{(4)}(0)\right)\right)-6 c_{1}^{3} c_{2}\left(20 c_{3}\right. \\
& \left(6 c_{4}\left(-44+42 G(0)+42 H(0)-7 G^{\prime}(0)+2 H^{\prime}(0)\right)+c_{1}(26-24 G(0)\right. \\
& \left.\left.\left.\left.-24 H(0)+5 G^{\prime}(0)-2 H^{\prime}(0)\right) A^{(3)}(0)\right) c_{1}(-1+G(0)+H(0))\left(-240 c_{6}+c_{1} A^{(5)}(0)\right)\right)\right) e_{n}^{7}+O\left(e_{n}^{8}\right) \text {. }
\end{aligned}
$$


Table 1 Some typical forms of the weight functions in (2), $\omega \in \mathbb{R}$

\begin{tabular}{llll}
\hline Methods & $A(\delta)$ & $G(t)$ & $H(\mu)$ \\
\hline Forms & $1+\delta^{3}+\omega \delta^{5}$ & $t^{2}+\omega t^{4}$ & $1+\mu+\omega \mu^{2}$ \\
\hline
\end{tabular}

By choosing $|G(0)|<\infty, G^{\prime}(0)=0, G^{\prime \prime}(0)=2, G^{(3)}(0)=0,\left|G^{(4)}(0)\right|<\infty$, $H(0)=1-G(0), H^{\prime}(0)=1$ and $\left|H^{\prime \prime}(0)\right|<\infty$, the error equations in the last step will be attained as comes next $e_{n+1}=\frac{1}{24 c_{1}^{7}} c_{2}\left(-2 c_{2}^{2}+c_{1} c_{3}\right)\left(24 c_{1} c_{2}^{2} c_{3}(7-\right.$ $\left.2 H^{\prime \prime}(0)\right)+12 c_{1}^{2} c_{3}^{2}\left(-2+H^{\prime \prime}(0)\right)+12 c_{1}^{2} c_{2}\left(-2 c_{4}+c_{1} A^{(3)}(0)\right)+c_{2}^{4}\left(-120+48 H^{\prime \prime}(0)+\right.$ $\left.\left.G^{(4)}(0)\right)\right) e_{n}^{8}+O\left(e_{n}^{9}\right)$. This shows that the iterative scheme $(2)$ can attain at least eighth order of convergence. Hence, the proof is complete.

Remark 1. The suggested class (2) includes only four evaluations per full cycle to achieve the local convergence order eight. Hence, its classical efficiency index is $8^{\frac{1}{4}} \approx 1.682$, which is much better than the efficiency of its origin, i.e. $6^{\frac{1}{4}} \approx 1.565$ of the method $(1)$.

Remark 2. The weight function $A(\delta)$ at the end of the first step gives a generality to this step. Since, its third derivative around zero has a clear effect on the final error equation (3). Such an effect provides a more generality to the proposed scheme along with furnishing a better condition for making with memory schemes out to (2).

\section{Some optimal concrete methods}

Some new methods can now be constructed using Theorem 1 and the class (2). Note that some typical forms of the weight functions satisfying Theorem 1 are illustrated in Table 1 . By applying $A^{(3)}(0)=0, H^{\prime \prime}(0)=0$, and $G^{(4)}(0)=120$ we have, for instance

$$
\left\{\begin{array}{l}
y_{n}=x_{n}-\frac{f\left(x_{n}\right)}{f^{\prime}\left(x_{n}\right)}\left(1+\left(\frac{f\left(x_{n}\right)}{f^{\prime}\left(x_{n}\right)}\right)^{5}\right), \\
z_{n}=y_{n}-\frac{f\left(y_{n}\right)}{f^{\prime}\left(x_{n}\right)}\left(1-\frac{f\left(y_{n}\right)}{f\left(x_{n}\right)}\right)^{-2}, \\
x_{n+1}=z_{n}-\frac{f\left(z_{n}\right)}{f^{\prime}\left(x_{n}\right)} \frac{\left(1+\left(\frac{f\left(y_{n}\right)}{f\left(x_{n}\right)}\right)^{2}+5\left(\frac{f\left(y_{n}\right)}{f\left(x_{n}\right)}\right)^{4}+\frac{f\left(z_{n}\right)}{f\left(y_{n}\right)}\right)}{\left(1-\frac{f\left(y_{n}\right)}{f\left(x_{n}\right)}-\frac{f\left(z_{n}\right)}{f\left(x_{n}\right)}\right)^{2}},
\end{array}\right.
$$

with the following error equation

$$
e_{n+1}=-\frac{c_{2}\left(-2 c_{2}^{2}+c_{1} c_{3}\right)\left(-7 c_{2}^{2} c_{3}+c_{1} c_{3}^{2}+c_{1} c_{2} c_{4}\right)}{c_{1}^{6}} e_{n}^{8}+O\left(e_{n}^{9}\right)
$$


By using $A^{(3)}(0)=6, H^{\prime \prime}(0)=0$, and $G^{(4)}(0)=120$, we have

$$
\left\{\begin{array}{l}
y_{n}=x_{n}-\frac{f\left(x_{n}\right)}{f^{\prime}\left(x_{n}\right)}\left(1+\left(\frac{f\left(x_{n}\right)}{f^{\prime}\left(x_{n}\right)}\right)^{3}\right), \\
z_{n}=y_{n}-\frac{f\left(y_{n}\right)}{f^{\prime}\left(x_{n}\right)}\left(1-\frac{f\left(y_{n}\right)}{f\left(x_{n}\right)}\right)^{-2}, \\
x_{n+1}=z_{n}-\frac{f\left(z_{n}\right)}{f^{\prime}\left(x_{n}\right)} \frac{\left(1+\left(\frac{f\left(y_{n}\right)}{f\left(x_{n}\right)}\right)^{2}+5\left(\frac{f\left(y_{n}\right)}{f\left(x_{n}\right)}\right)^{4}+\frac{f\left(z_{n}\right)}{f\left(y_{n}\right)}\right)}{\left(1-\frac{f\left(y_{n}\right)}{f\left(x_{n}\right)}-\frac{f\left(z_{n}\right)}{f\left(x_{n}\right)}\right)^{2}},
\end{array}\right.
$$

where its error relation reads

$$
e_{n+1}=\frac{c_{2}\left(-2 c_{2}^{2}+c_{1} c_{3}\right)\left(3 c_{1}^{2} c_{2}+7 c_{2}^{2} c_{3}-c_{1}\left(c_{3}^{2}+c_{2} c_{4}\right)\right)}{c_{1}^{6}} e_{n}^{8}+O\left(e_{n}^{9}\right) .
$$

Finally, by applying $A^{(3)}(0)=0, H^{\prime \prime}(0)=0$, and $G^{(4)}(0)=0$, we attain

$$
\left\{\begin{array}{l}
y_{n}=x_{n}-\frac{f\left(x_{n}\right)}{f^{\prime}\left(x_{n}\right)}\left(1-\left(\frac{f\left(x_{n}\right)}{f^{\prime}\left(x_{n}\right)}\right)^{5}\right) \\
z_{n}=y_{n}-\frac{f\left(y_{n}\right)}{f^{\prime}\left(x_{n}\right)}\left(1-\frac{f\left(y_{n}\right)}{f\left(x_{n}\right)}\right)^{-2} \\
x_{n+1}=z_{n}-\frac{f\left(z_{n}\right)}{f^{\prime}\left(x_{n}\right)} \frac{\left(1+\left(\frac{f\left(y_{n}\right)}{f\left(x_{n}\right)}\right)^{2}+\frac{f\left(z_{n}\right)}{f\left(y_{n}\right)}\right)}{\left(1-\frac{f\left(y_{n}\right)}{f\left(x_{n}\right)}-\frac{f\left(z_{n}\right)}{f\left(x_{n}\right)}\right)^{2}}
\end{array}\right.
$$

where its error equation is

$$
e_{n+1}=\frac{c_{2}\left(2 c_{2}^{2}-c_{1} c_{3}\right)\left(5 c_{2}^{4}-7 c_{1} c_{2}^{2} c_{3}+c_{1}^{2} c_{3}^{2}+c_{1}^{2} c_{2} c_{4}\right)}{c_{1}^{7}} e_{n}^{8}+O\left(e_{n}^{9}\right) .
$$

\section{Numerical examples}

In this section, we check the effectiveness of the iterative class (2) by choosing their members (11), (13), and (15). Due to this, we have compared them with the following scheme, described in [9]

$$
\left\{\begin{array}{l}
y_{n}=x_{n}-\frac{f\left(x_{n}\right)}{f^{\prime}\left(x_{n}\right)}, \\
z_{n}=x_{n}-\frac{f\left(x_{n}\right)}{f^{\prime}\left(x_{n}\right)} \frac{f\left(x_{n}\right)-f\left(y_{n}\right)}{f\left(x_{n}\right)-2 f\left(y_{n}\right)}, \\
x_{n+1}=u_{n}-\frac{3(\alpha+\beta)\left(u_{n}-z_{n}\right)}{\gamma\left(u_{n}-z_{n}\right)+\alpha\left(y_{n}-x_{n}\right)+\beta\left(z_{n}-x_{n}\right)} \frac{f\left(z_{n}\right)}{f^{\prime}\left(x_{n}\right)},
\end{array}\right.
$$

where $\alpha, \beta, \gamma \in \mathbb{R}, \alpha+\beta \neq 0$ and $u_{n}=z_{n}-\left(\frac{f\left(x_{n}\right)-f\left(y_{n}\right)}{f\left(x_{n}\right)-2 f\left(y_{n}\right)}+\frac{1}{2} \frac{f\left(z_{n}\right)}{f\left(y_{n}\right)-2 f\left(z_{n}\right)}\right)^{2} \frac{f\left(z_{n}\right)}{f^{\prime}\left(x_{n}\right)}$. We will choose $\alpha=\beta=\gamma=1$, in Table 3 .

The following optimal three-step eighth-order iteration [11] will also be used

$$
\left\{\begin{aligned}
y_{n}= & x_{n}-\frac{f\left(x_{n}\right)}{f^{\prime}\left(x_{n}\right)}, \\
z_{n}= & y_{n}-\frac{f\left(y_{n}\right)}{f^{\prime}\left(x_{n}\right)}\left\{1+2 \frac{f\left(y_{n}\right)}{f\left(x_{n}\right)}+4\left(\frac{f\left(y_{n}\right)}{f\left(x_{n}\right)}\right)^{2}\right\} \\
x_{n+1}= & z_{n}-\left\{\left(\frac{f\left(y_{n}\right)}{f\left(x_{n}\right)}\right)^{4}+\left(\frac{f\left(y_{n}\right)}{f\left(x_{n}\right)}\right)^{5}\right\} \frac{f\left(z_{n}\right)}{f^{\prime}\left(x_{n}\right)} \\
& \quad-\left\{1+\frac{f\left(z_{n}\right)}{f\left(x_{n}\right)}+\left(\frac{f\left(z_{n}\right)}{f\left(x_{n}\right)}\right)^{2}\right\} \frac{f\left[x_{n}, y_{n}\right] f\left(z_{n}\right)}{f\left[x_{n}, z_{n}\right] f\left[y_{n}, z_{n}\right]} .
\end{aligned}\right.
$$


For comparisons, we have also used the following methods presented in [14]

$$
\left\{\begin{array}{l}
y_{n}=x_{n}-\frac{f\left(x_{n}\right)}{f^{\prime}\left(x_{n}\right)}, \\
z_{n}=y_{n}-\frac{f\left(y_{n}\right)}{f^{\prime}\left(x_{n}\right)}\left\{\frac{2 f\left(x_{n}\right)-f\left(y_{n}\right)}{2 f\left(x_{n}\right)-5 f\left(y_{n}\right)}\right\}, \\
x_{n+1}=z_{n}-\frac{f\left(z_{n}\right)}{f^{\prime}\left(x_{n}\right)}\left\{\frac{2 f\left(x_{n}\right)-f\left(y_{n}\right)}{2 f\left(x_{n}\right)-5 f\left(y_{n}\right)}\right\}\left\{1+2 \frac{f\left(z_{n}\right)}{f\left(x_{n}\right)}+\frac{f\left(z_{n}\right)}{f\left(y_{n}\right)}\right. \\
\left.+\left(\frac{f\left(z_{n}\right)}{f\left(y_{n}\right)}\right)^{2}+\left(\frac{f\left(y_{n}\right)}{f\left(x_{n}\right)}\right)^{2}+4\left(\frac{f\left(y_{n}\right)}{f\left(x_{n}\right)}\right)^{3}+12\left(\frac{f\left(y_{n}\right)}{f\left(x_{n}\right)}\right)^{4}-\frac{f\left(z_{n}\right)}{f^{\prime}\left(x_{n}\right)}-\left(\frac{f\left(y_{n}\right)}{f^{\prime}\left(x_{n}\right)}\right)^{2}\right\},
\end{array}\right.
$$

and

$$
\left\{\begin{array}{l}
y_{n}=x_{n}-\frac{f\left(x_{n}\right)}{f^{\prime}\left(x_{n}\right)}, \\
z_{n}=y_{n}-\frac{f\left(y_{n}\right)}{f^{\prime}\left(x_{n}\right)}\left\{\frac{f\left(x_{n}\right)}{f\left(x_{n}\right)-2 f\left(y_{n}\right)}\right\} \\
x_{n+1}=z_{n}-\frac{f\left(z_{n}\right)}{f^{\prime}\left(x_{n}\right)}\left\{\frac{f\left(x_{n}\right)}{f\left(x_{n}\right)-2 f\left(y_{n}\right)}\right\}\left\{1+2 \frac{f\left(z_{n}\right)}{f\left(x_{n}\right)}+\frac{f\left(z_{n}\right)}{f\left(y_{n}\right)}+\left(\frac{f\left(y_{n}\right)}{f\left(x_{n}\right)}\right)^{2}\right. \\
\left.+2\left(\frac{f\left(y_{n}\right)}{f\left(x_{n}\right)}\right)^{3}-\frac{f\left(z_{n}\right)}{f^{\prime}\left(x_{n}\right)}+\left(\frac{f\left(y_{n}\right)}{f^{\prime}\left(x_{n}\right)}\right)^{3}\right\} .
\end{array}\right.
$$

The nonlinear test functions are furnished in Table 2.

Table 2 Test functions and their zeros

\begin{tabular}{ll}
\hline Test Nonlinear Functions & Simple Zeros \\
\hline$f_{1}(x)=10 x e^{-x^{2}}-1$ & $\alpha_{1} \approx 1.6796306104284499406749203388379703$ \\
$f_{2}(x)=x^{5}+x^{4}+4 x^{2}-15$ & $\alpha_{2} \approx 1.3474280989683049815067153807148212$ \\
$f_{3}(x)=x e^{2}-(\sin (x))^{2}+3 \cos (x)+5$ & $\alpha_{3} \approx-1.20764782713091892700941675835608$ \\
$f_{4}(x)=x^{4}+\sin \left(\frac{\pi}{x^{2}}\right)-5$ & $\alpha_{4}=\sqrt{2}$ \\
$f_{5}(x)=x^{2} e^{x}-\sin (x)$ & $\alpha_{5}=0$ \\
$f_{6}(x)=\left(\sin (x)-\frac{\sqrt{2}}{2}\right)^{2}(x+1)$ & $\alpha_{6}=-1$ \\
$f_{7}(x)=11 x^{11}-1$ & $\alpha_{7} \approx 0.8041330975036643237414634983732973$ \\
$f_{8}(x)=\sin (3 x)+x \cos (x)$ & $\alpha_{8} \approx 1.1977695352162711659385794729509898$ \\
\hline
\end{tabular}

The results of comparisons are given in Table 3 in terms of the number significant digits for each test function after 3 iterations (with the same Total Number of Evaluations; TNE=12), that is, e.g. $0.1 e-680$ shows that the absolute value of the given nonlinear function $\left(f_{1}\right)$ after three iterations is zero up to 680 decimal places. For numerical comparisons, the stopping criterion is $\left|f\left(x_{n}\right)\right|<1 . E-1000$. We used MatLaB 7.5 for numerical computations and variable precision arithmetics with 1100 digits of mantissa.

Although the results shown in Table 3 are not as good as would be desirable for methods (11), (13) and (15), the dynamical study that we show below indicates that there are important aspects in which new methods improve other eighth-order known methods. We are going to study the basins of attraction of the different methods for finding the roots of unity.

There are various criteria involved in choosing an iterative method to approximate the root of an equation. These include the initial value problem, the rate of convergence and the complexity of the calculation. In order to investigate these dynamics with some higher order methods we have improved the method (1) and now study their complex dynamics. 
Table 3 Comparison of different methods for solving test functions with $T N E=12$.

\begin{tabular}{|c|c|c|c|c|c|c|c|c|c|}
\hline$f$ & Guess & & $(17)$ & (18) & (19) & $(20)$ & (11) & (13) & $(15)$ \\
\hline \multirow[t]{3}{*}{$f_{1}$} & 1.72 & \multirow[t]{3}{*}{$|f|$} & $0.1 \mathrm{e}-680$ & $0.6 \mathrm{e}-663$ & $0.3 \mathrm{e}-765$ & $0.5 \mathrm{e}-659$ & $0.2 \mathrm{e}-660$ & $0.6 \mathrm{e}-700$ & $0.3 e-654$ \\
\hline & 1 & & $0.3 \mathrm{e}-118$ & $0.4 \mathrm{e}-221$ & $0.3 \mathrm{e}-231$ & $0.4 \mathrm{e}-167$ & $0.5 \mathrm{e}-213$ & $0.8 \mathrm{e}-53$ & $0.6 \mathrm{e}-86$ \\
\hline & 1.5 & & $0.2 \mathrm{e}-391$ & $0.4 \mathrm{e}-473$ & $0.2 \mathrm{e}-438$ & $0.1 \mathrm{e}-376$ & $0.2 \mathrm{e}-375$ & $0.5 \mathrm{e}-366$ & $0.8 \mathrm{e}-361$ \\
\hline \multirow[t]{3}{*}{$f_{2}$} & 1 & \multirow[t]{3}{*}{$|f|$} & $0.1 \mathrm{e}-190$ & - & $0.1 \mathrm{e}-49$ & $0.2 \mathrm{e}-127$ & $0.4 \mathrm{e}-80$ & $0.2 \mathrm{e}-132$ & $0.9 \mathrm{e}-197$ \\
\hline & 1.1 & & $0.5 e-278$ & $0.1 \mathrm{e}-81$ & $0.2 \mathrm{e}-168$ & $0.7 \mathrm{e}-223$ & $0.7 \mathrm{e}-165$ & $0.2 \mathrm{e}-197$ & $0.5 e-299$ \\
\hline & 1.8 & & $0.1 \mathrm{e}-213$ & $0.1 \mathrm{e}-215$ & $0.2 \mathrm{e}-227$ & $0.5 \mathrm{e}-194$ & $0.1 \mathrm{e}-195$ & $0.2 \mathrm{e}-189$ & $0.4 \mathrm{e}-187$ \\
\hline \multirow[t]{3}{*}{$f_{3}$} & -1.1 & \multirow[t]{3}{*}{$|f|$} & $0.1 e-516$ & $0.6 \mathrm{e}-292$ & $0.3 \mathrm{e}-322$ & $0.6 \mathrm{e}-423$ & $0.1 \mathrm{e}-325$ & $0.1 \mathrm{e}-325$ & $0.6 e-433$ \\
\hline & -1.5 & & $0.1 \mathrm{e}-253$ & $0.4 \mathrm{e}-238$ & $0.5 \mathrm{e}-187$ & $0.1 \mathrm{e}-216$ & $0.2 \mathrm{e}-253$ & $0.1 \mathrm{e}-247$ & $0.2 \mathrm{e}-205$ \\
\hline & -1 & & $0.3 e-379$ & $0.1 \mathrm{e}-73$ & $0.8 \mathrm{e}-151$ & $0.5 e-269$ & $0.4 \mathrm{e}-158$ & $0.1 \mathrm{e}-143$ & $0.4 \mathrm{e}-254$ \\
\hline \multirow[t]{3}{*}{$f_{4}$} & 1.3 & \multirow[t]{3}{*}{$|f|$} & $0.3 \mathrm{e}-470$ & $0.5 \mathrm{e}-439$ & $0.4 \mathrm{e}-439$ & $0.1 \mathrm{e}-507$ & $0.3 e-504$ & $0.1 \mathrm{e}-459$ & $0.3 e-528$ \\
\hline & 1 & & $0.3 e-232$ & $0.2 \mathrm{e}-269$ & $0.6 \mathrm{e}-263$ & $0.2 \mathrm{e}-289$ & $0.2 \mathrm{e}-199$ & $0.2 \mathrm{e}-146$ & $0.5 \mathrm{e}-227$ \\
\hline & 1.6 & & $0.2 \mathrm{e}-469$ & $0.1 \mathrm{e}-363$ & $0.3 e-394$ & $0.8 \mathrm{e}-447$ & $0.3 \mathrm{e}-376$ & $0.3 \mathrm{e}-351$ & $0.5 \mathrm{e}-430$ \\
\hline \multirow[t]{3}{*}{$f_{5}$} & 0.1 & \multirow[t]{3}{*}{$|f|$} & $0.8 \mathrm{e}-360$ & $0.5 \mathrm{e}-350$ & $0.1 \mathrm{e}-393$ & $0.1 \mathrm{e}-343$ & $0.6 \mathrm{e}-349$ & $0.1 \mathrm{e}-357$ & $0.1 \mathrm{e}-338$ \\
\hline & 0.5 & & $0.6 \mathrm{e}-400$ & $0.1 \mathrm{e}-317$ & $0.1 \mathrm{e}-385$ & $0.3 \mathrm{e}-366$ & $0.2 \mathrm{e}-342$ & $0.8 \mathrm{e}-342$ & $0.8 \mathrm{e}-382$ \\
\hline & -0.1 & & $0.3 e-476$ & $0.5 \mathrm{e}-482$ & $0.3 e-476$ & $0.8 \mathrm{e}-457$ & $0.4 \mathrm{e}-441$ & $0.6 \mathrm{e}-438$ & $0.2 \mathrm{e}-436$ \\
\hline \multirow[t]{3}{*}{$f_{6}$} & -0.8 & \multirow[t]{3}{*}{$|f|$} & $0.2 \mathrm{e}-294$ & $0.1 \mathrm{e}-212$ & $0.9 \mathrm{e}-398$ & $0.5 \mathrm{e}-274$ & $0.2 \mathrm{e}-248$ & $0.7 \mathrm{e}-242$ & $0.3 e-288$ \\
\hline & -1.2 & & $0.1 \mathrm{e}-441$ & $0.1 \mathrm{e}-421$ & $0.4 \mathrm{e}-421$ & $0.2 \mathrm{e}-402$ & $0.1 \mathrm{e}-385$ & $0.2 \mathrm{e}-380$ & $0.5 e-381$ \\
\hline & -0.9 & & $0.2 \mathrm{e}-483$ & $0.1 \mathrm{e}-498$ & $0.1 \mathrm{e}-553$ & $0.6 \mathrm{e}-469$ & $0.2 \mathrm{e}-500$ & $0.1 \mathrm{e}-454$ & $0.2 \mathrm{e}-457$ \\
\hline \multirow[t]{3}{*}{$f_{7}$} & 0.81 & \multirow[t]{3}{*}{$|f|$} & $0.5 \mathrm{e}-752$ & $0.5 e-695$ & $0.4 \mathrm{e}-737$ & $0.8 \mathrm{e}-717$ & $0.4 \mathrm{e}-697$ & $0.5 \mathrm{e}-697$ & $0.5 e-716$ \\
\hline & 0.79 & & $0.5 e-546$ & $0.3 e-461$ & $0.2 \mathrm{e}-485$ & $0.3 e-503$ & $0.1 \mathrm{e}-460$ & $0.2 \mathrm{e}-460$ & $0.5 e-519$ \\
\hline & 1 & & $0.5 \mathrm{e}-76$ & $0.3 e-62$ & $0.1 \mathrm{e}-106$ & $0.3 \mathrm{e}-65$ & $0.5 \mathrm{e}-64$ & $0.6 \mathrm{e}-64$ & $0.2 \mathrm{e}-61$ \\
\hline \multirow[t]{3}{*}{$f_{8}$} & 1 & \multirow[t]{3}{*}{$|f|$} & $0.2 \mathrm{e}-372$ & $0.5 e-589$ & $0.1 \mathrm{e}-435$ & $0.5 e-434$ & $0.2 \mathrm{e}-378$ & $0.2 \mathrm{e}-605$ & $0.7 e-374$ \\
\hline & 0.8 & & $0.1 \mathrm{e}-150$ & $0.1 \mathrm{e}-91$ & $0.2 \mathrm{e}-160$ & $0.7 \mathrm{e}-156$ & $0.8 \mathrm{e}-159$ & $0.4 \mathrm{e}-193$ & $0.2 \mathrm{e}-116$ \\
\hline & 1.5 & & $0.1 \mathrm{e}-188$ & $0.2 \mathrm{e}-102$ & $0.9 \mathrm{e}-194$ & $0.6 \mathrm{e}-175$ & $0.2 \mathrm{e}-147$ & $0.1 \mathrm{e}-121$ & $0.3 e-224$ \\
\hline
\end{tabular}

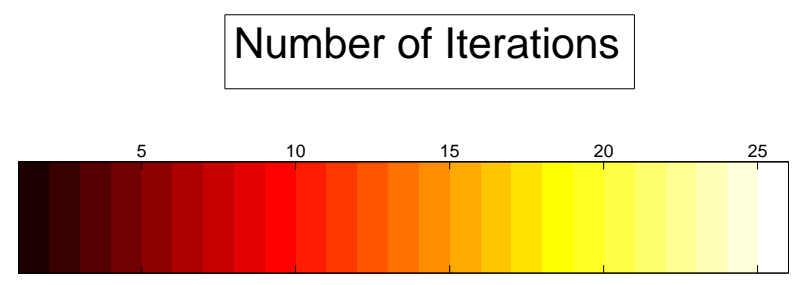

Fig. 1 HOT Colormap in MATLAB

Note that a point $z_{0}$ belongs to the Julia set if and only if dynamics in a neighborhood of $z_{0}$ displays sensitive dependence on the initial conditions, so that nearby initial conditions lead to wildly different behavior after a number of iterations. 


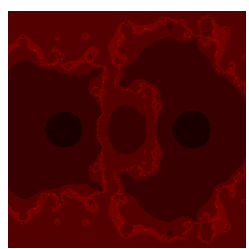

(a) (11)

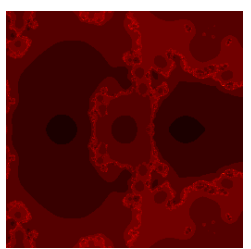

(b) (13)

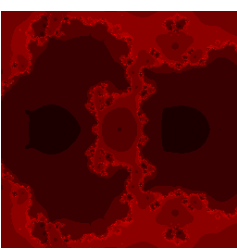

(c) (15)

Fig. 2 Basins of attraction of methods (11), (13) and (15) for $f(x)=x^{2}-1$

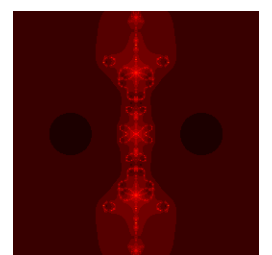

(a) (17)

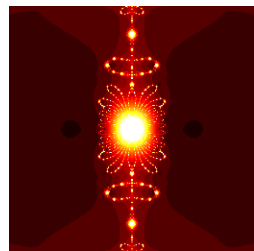

(b) (18)

Fig. 3 Basins of attraction of methods (17) and (18) for $f(x)=x^{2}-1$

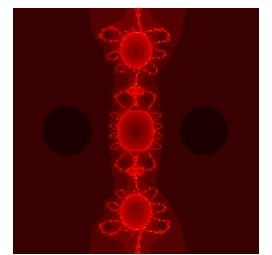

(a) (19)

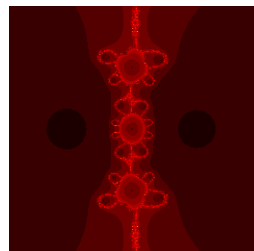

(b) (20)

Fig. 4 Basins of attraction of methods (19) and (20) for $f(x)=x^{2}-1$

5 Fractal pictures for the basins of attractions of the eighth order methods

We consider the polynomial $f(x)=x^{r}-1, x \in \mathbb{C}$ for finding the $r$ th roots of unity. The $r$ th roots of unity are given by

$$
\alpha_{j}=\cos \left(\frac{2 \pi(j-1)}{r}\right)+i \sin \left(\frac{2 \pi(j-1)}{r}\right), j=1,2 \ldots r .
$$

The basin of attraction corresponding to a zero $\alpha_{j}$ of the polynomial $f(x)$ is the set of all starting points $x_{0}$ which are attracted to $\alpha_{j}$. We use a similar like technique as in [23] to generate the basins of attraction.

In our numerical experiments, we take a square $\mathbb{R} \times \mathbb{R}=[-2,2] \times[-2,2]$ of $256 \times 256$ points and we apply the eighth order iterative method starting in every $x_{0}$ in the square. 
If the sequence generated by iterative method attempt a zero $\alpha_{j}$ of the polynomial with a tolerance $\left|f\left(x_{k}\right)\right|<1 e-5$ and a maximum of 25 iterations, we decide that $x_{0}$ is in the basin of attraction of these zero.

If the iterative method starting in $x_{0}$ reaches a zero in $n$ iterations $(n \leq 25)$, then we mark this point $x_{0}$ with a color depending on $n$ with colormap hot in MATLAB as shown in Figure 1. If $n>25$, we conclude that the starting point has diverged and we assign a white color. Let us denote $\mu$ be the mean number of iterations for the converging points and $N_{d}$ the number of diverging points. We choose $r=2,3,7,20$ for our numerical experiments.

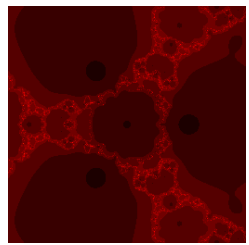

(a) (11)

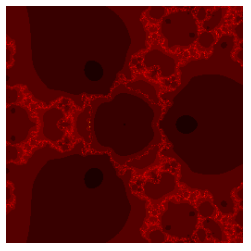

(b) (13)

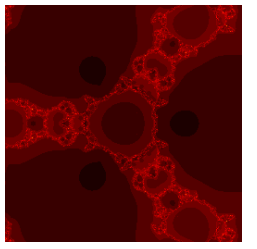

(c) (15)

Fig. 5 Basins of attraction of methods (11), (13) and (15) for $f(x)=x^{3}-1$

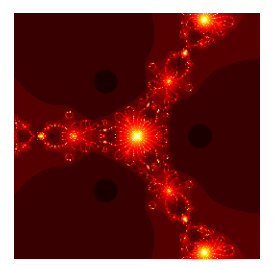

(a) (17)

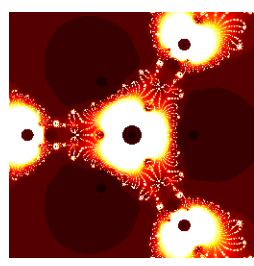

(b) (18)

Fig. 6 Basins of attraction of methods (17) and (18) for $f(x)=x^{3}-1$

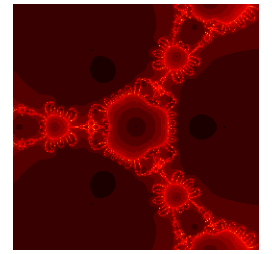

(a) (19)

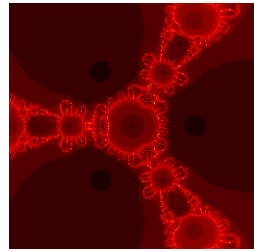

(b) (20)

Fig. 7 Basins of attraction of methods (19) and (20) for $f(x)=x^{3}-1$ 


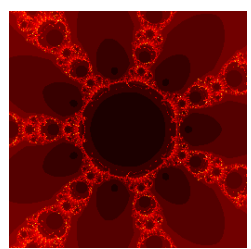

(a) (11)

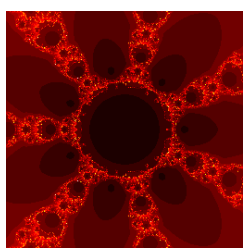

(b) (13)

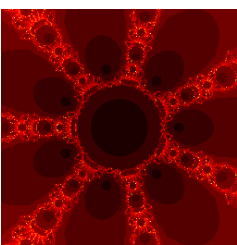

(c) (15)

Fig. 8 Basins of attraction of methods (11), (13) and (15) for $f(x)=x^{7}-1$

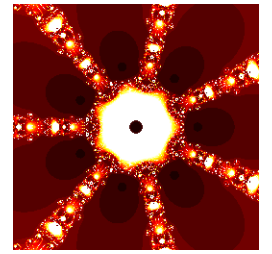

(a) (17)

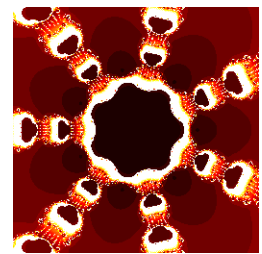

(b) (18)

Fig. 9 Basins of attraction of methods (17) and (18) for $f(x)=x^{7}-1$

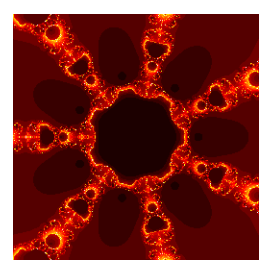

(a) (19)

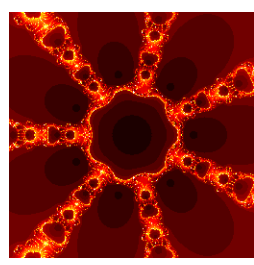

(b) (20)

Fig. 10 Basins of attraction of methods (19) and (20) for $f(x)=x^{7}-1$

Table 4 gives a comparison of $\mu$ and $N_{d}$ of the eighth order methods for the polynomials $f(x)=x^{r}-1, r=2,3,7,20$. We also show a column with the percentage of white points for each method. We can observe that all methods (except (18)) are globally convergent for quadratic polynomials, being the mean number of iterations similar in all cases. Proposed methods are also globally convergent for cubic polynomials as well as (19) and (20). Although, the global convergence is missed for higher degree of the polynomials, the number of divergent points is much lower in new methods than in known ones. 


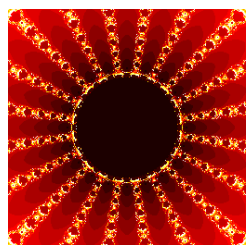

(a) (11)

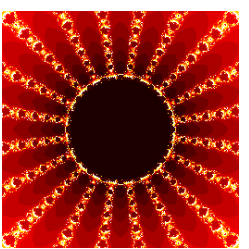

(b) (13)

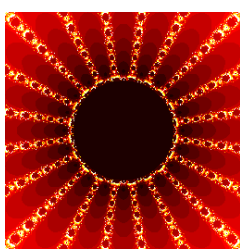

(c) $(15)$

Fig. 11 Basins of attraction of methods (11), (13) and (15) for $f(x)=x^{20}-1$

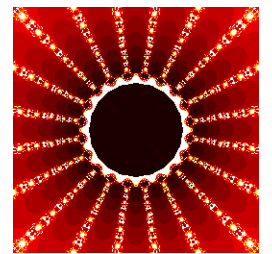

(a) $(17)$

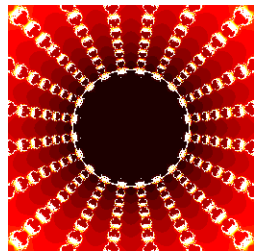

(b) (18)

Fig. 12 Basins of attraction of methods (17) and (18) for $f(x)=x^{20}-1$

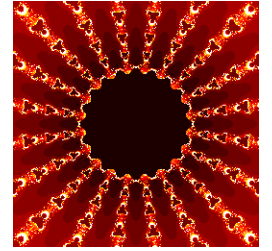

(a) (19)

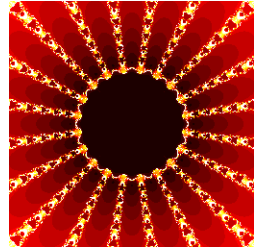

(b) (20)

Fig. 13 Basins of attraction of methods (19) and (20) for $f(x)=x^{20}-1$

Figures 2 (a)-(c) show the basins of attraction for our new methods (11), (13) and (15), respectively for the quadratic polynomial. The dark regions show the roots $\alpha_{1}=-1$ and $\alpha_{2}=1$. This shows the rapid convergence of eighth order methods when the starting points are chosen close to the root. There are no diverging points for the 3 methods and all methods converge in less than 10 iterations as shown by the dark intensity of the colors. Figures (3) (a)-(b) and (4) (a)-(b) show the basins of attraction for the methods (17)-(20), respectively. We observe that there are some diverging points for method (18) which is based on divided difference methods and is the worst method. We can observe the beautiful pictures produced by each method. The most efficient method with the lowest mean iteration number and greatest basins of attraction for the quadratic polynomial is (17). These figures confirm graphically the numerical results shown in Table 4. 
Table 4 Comparison of $\mu, N_{d}$ and $P_{d}$ of the eighth order methods for the polynomials $f(x)=x^{r}-1, r=2,3,7,20$.

\begin{tabular}{llllllllllllll}
\hline Method & \multicolumn{3}{c}{$r=2$} & \multicolumn{3}{c}{$r=3$} & \multicolumn{3}{c}{$r=7$} & \multicolumn{3}{c}{$r=20$} \\
\hline & $N_{d}$ & $P_{d}$ & $\mu$ & $N_{d}$ & $P_{d}$ & $\mu$ & $N_{d}$ & $P_{d}$ & $\mu$ & $N_{d}$ & $P_{d}$ & $\mu$ \\
$(11)$ & 0 & 0 & 2.61 & 0 & 0 & 2.63 & 0 & 0 & 3.50 & 1816 & 2.8 & 5.91 \\
$(13)$ & 0 & 0 & 2.92 & 0 & 0 & 2.88 & 2 & 0.003 & 3.63 & 2097 & 3.2 & 5.92 \\
$(15)$ & 0 & 0 & 3.09 & 0 & 0 & 2.72 & 4 & 0.006 & 3.51 & 1630 & 2.5 & 5.79 \\
$(17)$ & 0 & 0 & 2.34 & 66 & 0.1 & 3.12 & 6382 & 9.7 & 4.47 & 5380 & 8.2 & 5.34 \\
$(18)$ & 772 & 1.18 & 3.22 & 10534 & 16.1 & 4.58 & 8768 & 13.4 & 4.57 & 7140 & 10.9 & 5.98 \\
$(19)$ & 0 & 0 & 2.58 & 0 & 0 & 3.08 & 72 & 0.1 & 3.86 & 2792 & 4.3 & 4.93 \\
$(20)$ & 0 & 0 & 2.53 & 0 & 0 & 3.16 & 160 & 0.2 & 4.35 & 3418 & 5.2 & 5.87 \\
\hline
\end{tabular}

Figures 5, 6 and 7 show the basins of attractions for the cubic polynomial. There are some diverging points for method (17) but a lot for method (18). The other methods are globally convergent. The best method in terms of lowest mean iteration number (see Table 4) and largest basins of attraction is method (11).

As the degree of the polynomial increases from 3 to 7 , the methods have difficulties and their mean iteration number increases. Our methods have very little diverging points compared to the other ones. Method (11) is globally convergent. Figures 8, 9 and 10 show the basins of attractions for finding the 7 th roots of unity. The fractal of the methods (11), (13) and (15) are almost similar. We also observe in Figure 9 methods (17) and (18) have a lot of white (divergent) points which is confirmed by Table 4 . The method (11) is again the most efficient method.

For a large $r=20$, the complexity of the fractals increases for the methods as shown as in Figures 11, 12 and 13. The fractals are almost similar and the number of diverging points are high for all methods. Method (15) has the lowest number of diverging points whereas method (19) has the lowest mean iteration number of the converging points. On the whole, we see that our methods are better than the other methods in the literature.

\section{Conclusion}

Our main goal and motivation in constructing iterative methods for solving nonlinear equations is to attain as high as possible order of convergence with minimal computational cost. The most efficient existing root solvers are based on multi-point iterations, first studied in the Traub's book [24]; and recently have been analyzed in the Ph.D. Thesis [25].

A novel eighth-order class of derivative-involved methods without memory including three steps and four functional evaluations per full cycle to reach the 
optimal convergence order 8 have developed by using the method of Chun and Neta (1), and weight function technique. The contributed class has arrived at the optimal efficiency index $8^{\frac{1}{4}} \approx 1.682$. The convergence analysis of $(2)$ has been made. Finally, numerical examples were employed to support the theory presented in this paper. The basins of attraction of the new methods have also been provided and they have shown that the new methods can compete with other optimal eighth order methods in the literature.

Acknowledgements The authors would like to thank the referees for the valuable comments and for the suggestions to improve the readability of the paper.

\section{References}

1. J.S. Pang, D. Chan, Iterative methods for variational and complementary problems, Mathematical Programming, 24(1), 284-313, (1982).

2. D. Sun, A class of iterative methods for solving nonlinear projection equations, Journal of Optimization Theory and Applications, 91(1), 123-140, (1996)

3. C. Chun, B. Neta, A new sixth-order scheme for nonlinear equations, Appl. Math. Lett. $25,185-189,(2012)$.

4. H.T. Kung, J.F. Traub, Optimal order of one-point and multipoint iteration, J. ACM, 21, 643-651, (1974).

5. B. Neta, A new family of high-order methods for solving equations, International Journal of Computer Mathematics, 14, 191-195, (1983).

6. B. Neta, On Popovski's method for nonlinear equations, Applied Mathematics and Computation, 201, 710-715, (2008).

7. C. Chun, B. Neta, Some modifications of Newton's method by the method of undeterminate coefficients, Computers and Mathematics with Applications, 56, 2528-2538, (2008).

8. C. Chun, M.Y. Lee, B. Neta, J. Dzunic, On optimal fourth-order iterative methods free from second derivative and their dynamics, Applied Mathematics and Computation, 218, 6427-6438, (2012).

9. A. Cordero, J.R. Torregrosa, M.P. Vassileva, Three-step iterative methods with optimal eighth-order convergence, J. Comput. Appl. Math., 235 , 3189-3194, (2011).

10. A. Cordero, J.R. Torregrosa, M.P. Vassileva, A family of modified Ostrowski's methods with optimal eighth order of convergence, Appl. Math. Lett., 24, 2082-2086 (2011).

11. M. Heydari, S.M. Hosseini, G.B. Loghmani, On two new families of iterative methods for solving nonlinear equations with optimal order, Appl. Anal. Dis. Math., 5, 93-109, (2011).

12. B. Neta, M.S. Petkovic, Construction of optimal order nonlinear solvers using inverse interpolation, Applied Mathematics and Computation, 217, 2448-2445, (2010).

13. M. Sharifi, D.K.R. Babajee, F. Soleymani, Finding the solution of nonlinear equations by a class of optimal methods, Comput. Math. Appl., 63, 764-774, (2012).

14. F. Soleymani, S. Karimi Vanani, M. Khan, M. Sharifi, Some modifications of King's family with optimal eighth order of convergence, Mathematical Computer Modelling 55, 1373-1380, (2012).

15. F. Soleymani, S. Karimi Vanani, M. Jamali Paghaleh, A class of three-step derivativefree root solvers with optimal convergence order, J. Appl. Math., Volume 2012, Article ID 568740, 15 pages. doi:10.1155/2012/568740.

16. F. Soleymani, M. Sharifi, B.S. Mousavi, An improvement of Ostrowski's and King's techniques with optimal convergence order eight, Journal of Optimization Theory and Applications, 153, 225-236, (2012).

17. B.D. Stewart, Attractor bassins of various root-finding methods, M.S. Thesis, Naval Postgraduate School, Department of Applied Mathematics, Monterey CA, June 2001. 
18. S. Amat, S. Busquier, S. Plaza, Review of some iterative root-finding methods from a dynamical point of view, Scientia, 10, 3-35, (2004).

19. S. Amat, S. Busquier, S. Plaza, Dynamics of the King and Jarratt iterations, Aequationes Math., 69, 212-223, (2005).

20. S. Amat, S. Busquier, S. Plaza, Chaotic dynamics of a third-order Newton type method, J. Math. Anal. Appl., 366, 24-32, (2010).

21. B. Neta,C. Chun, M. Scott, A note on the modified super-Halley method, Applied Mathematics and Computation, 218, 9575-9577, (2012).

22. M. Scott, B. Neta, C. Chun, Basin attractors for various methods, Applied Mathematics and Computation, 218, 2584-2599, (2011).

23. G. Ardelean, A comparison between iterative methods by using the basins of attraction, Appl. Math. Comput., 218, 88-95, (2011)

24. J.F. Traub, Iterative Methods for the Solution of Equations. Prentice Hall, New York, (1964).

25. D.K.R. Babajee, Analysis of higher order variants of Newton's method and their applications to differential and integral equations and in ocean acidification, Ph.D. Thesis, University of Mauritius, December (2010). 